\title{
Parameterization of the duration of infection stages of serotype $O$ foot-and-mouth disease virus: an analytical review and meta-analysis with application to simulation models
}

\author{
Fernando Mardones ${ }^{1 *}$, Andrés Perez ${ }^{1,2 \dagger}$, Javier SAnChez ${ }^{3 \dagger}$, \\ Mohammad Alkhamis ${ }^{1,4}$, Tim CARPenter ${ }^{1}$ \\ ${ }^{1}$ Center for Animal Disease Modeling and Surveillance (CADMS), School of Veterinary Medicine, \\ University of California, Davis, CA 95616, USA \\ ${ }^{2}$ CONICET and Facultad de Ciencias Veterinarias UNR, Argentina \\ ${ }^{3}$ Department of Health Management, University of Prince Edward Island (UPEI), Charlottetown, Canada \\ ${ }^{4}$ Department of Aridland Agriculture and Greenery, Food Resources Division, \\ Kuwait Institute for Scientific Research, AlShuwikh, Kuwait
}

(Received 7 October 2009; accepted 4 March 2010)

\begin{abstract}
Foot-and-mouth disease (FMD) is considered one of the most important infectious diseases of livestock because of the devastating economic consequences that it inflicts in affected regions. The value of critical parameters, such as the duration of the latency or the duration of the infectious periods, which affect the transmission rate of the FMD virus (FMDV), are believed to be influenced by characteristics of the host and the virus. Disease control and surveillance strategies, as well as FMD simulation models, will benefit from improved parameter estimation. The objective of this study was to quantify the distributions of variables associated with the duration of the latency, subclinical, incubation, and infectiousness periods of FMDV transmission. A double independent, systematic review of 19 retrieved publications reporting results from experimental trials, using 295 animals in four reference laboratories, was performed to extract individual values related to FMDV transmission. Probability density functions were fitted to data and a set of regression models were used to identify factors associated with the assessed parameters. Latent, subclinical, incubation, and infectious periods ranged from 3.1 to $4.8,2$ to $2.3,5.5$ to 6.6 , and 3.3 to 5.7 days, respectively. Durations were significantly $(p<0.05)$ associated independently with route of exposure, type of donor, animal species, strains, characteristics of sampling, and clinical signs. These results will contribute to the improvement of disease control and surveillance strategies and stochastic models used to simulate FMD spread and, ultimately, development of cost-effective plans to prevent and control the potential spread of the disease in FMD-free regions of the world.
\end{abstract}

foot-and-mouth disease / individual data meta-analysis / frailty model / epidemiologic model / disease stage

\section{INTRODUCTION}

Foot-and-mouth disease (FMD) is a World Animal Health Organization (OIE)-listed

\footnotetext{
$\bar{\dagger}^{\dagger}$ Authors contributed equally.

* Corresponding author: fomardones@ucdavis.edu
}

disease that is considered one of the most highly contagious diseases of domestic and wild cloven-hoofed animals [30]. FMD is caused by a virus (FMDV) that belongs to the 


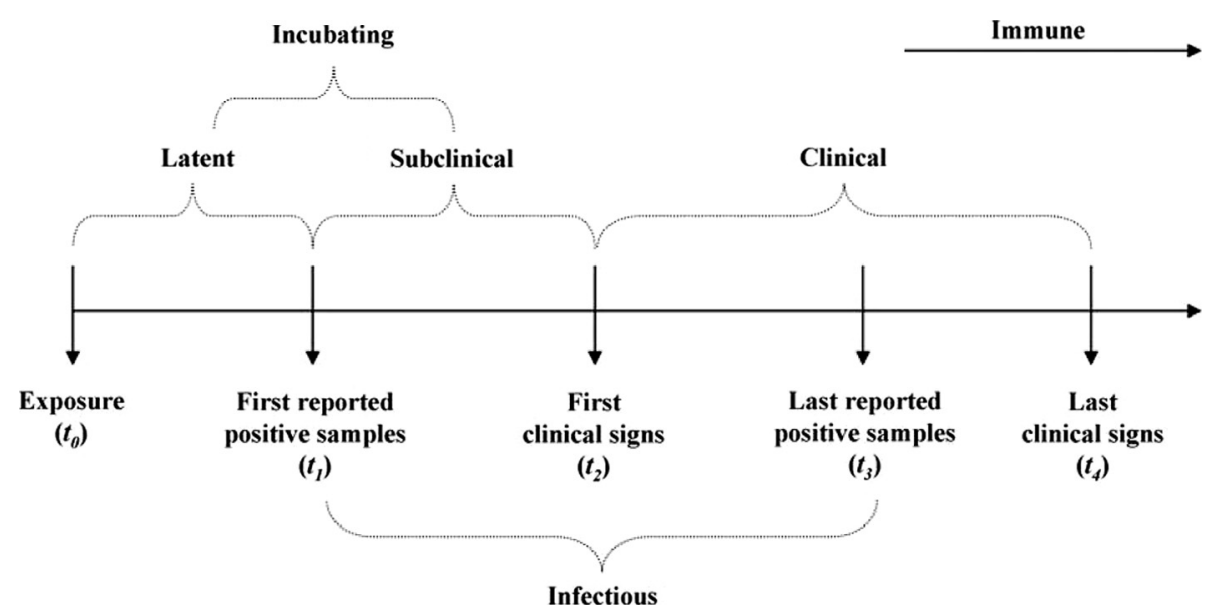

Figure 1. Potential stages of FMDV infection in susceptible animals after experimental exposure to an infected animal.

Picornaviridae family [7], which has been grouped into seven distinct serotypes, referred to as $\mathrm{A}, \mathrm{O}, \mathrm{C}$, Asia 1, and the three groups originally isolated in the southern African territories (SAT-1, SAT-2, and SAT-3). There is no cross immunological reaction between serotypes; subsequently, infection with a given serotype does not provide immunity against another.

Direct contact between susceptible and infectious animals and indirect transmission via contaminated products are the most common routes for FMDV transmission [5]. Airborne transmission has also been suggested, particularly when favorable environmental conditions take place [24].

FMDV infection is characterized by an acute febrile condition, with the development of vesicles in mouth, tongue, nose, feet, and udders. Reviews of the clinical variation of FMDV infection in livestock are available elsewhere [33-35]. Although FMD is characterized by a high morbidity rate, mortality is rare $(<5 \%)$ in adult animals. It has been hypothesized that disease severity varies with level of immunity, infectious dose, route of exposure, virus strain, environmental and animal species, but also within the same species, e.g. age and breed.

During and immediately after the 2001 UK FMD epidemic, there was a notable proliferation of epidemic models aimed to simulate the spread, predicting the impact, and identifying the most cost-effective strategies to control or prevent the consequences of the introduction of the FMDV in susceptible animal populations in FMD-free countries [32]. In general, the methodological approach used to model FMD spread is to divide the individuals into compartments using the susceptible-latent-infectiousresistant (or removed) framework (SLIR, Fig. 1) [4]. A susceptible animal (S) becomes infected after effectively contacting, directly or indirectly, an infectious animal or contaminated fomite; after a latent (L) period, the infected animal becomes infectious (I) and subsequently dies, becomes immune, or is culled as part of emergency control strategies (R). Additional compartments may be considered in the model to, for example, differentiate subclinical from clinical stages of infection, or to include the vaccinated status as a transitional stage.

Estimates of parameter values that are used to model the transition among compartments are often obtained by fitting the model to epidemic or historical data [31], eliciting expert opinion [6, 51], or using information emerging from experimental trials. Although these methods may provide satisfactory estimates for the models with a single or limited number of parameters, systematic reviews and metaanalysis of existing evidence will produce more 
precise estimates than those currently available, through the quantification of uncertainty and the identification of sources of variability for the parameters.

This systematic review of reported experimental trials with donor animals related to FMDV focused on the parameterization of serotype O FMDV transmission, because it is the most widely distributed and prevalent FMDV serotype [38]. Aims of the study were to estimate the most likely values for the FMD stage duration parameters and to quantify the strength of their association with epidemiologic factors hypothesized to influence their distribution. Results presented here will be useful for the parameterization of FMD spread models that will ultimately be used to evaluate control strategies of FMD in endemic and free regions, which may become infected.

\section{MATERIALS AND METHODS}

\subsection{Literature search}

Literature searches were conducted in English, Spanish, and Portuguese to identify experimental trials in which infection of livestock species with FMDV donor animals was reported. Two familiar electronic databases for the authors, ISI $^{1}$, and PubMed (MEDLINE ${ }^{2}$ ), were explored through the local server of the University of California at Davis (UCD) ${ }^{3}$ using the multiple keywords and expressions (pig* OR swine OR porcine OR hog OR sow OR cattle OR bovine OR cow OR bull OR steer OR calves OR heifer OR calf OR sheep OR goat OR domestic) AND (foot-and-mouth OR FMD* OR aphtho*) AND (infect* OR transm*) AND (exper* OR excret* OR secret*) AND (incubation OR latency $O R$ clinical $O R$ carrier). Because experimental trials often evaluate different FMDV serotypes, searches were not limited to serotype $\mathrm{O}$; however, were restricted to include publications from 1 January 1960 through 31 September 2007. References cited in retrieved reports were reviewed to identify additional reports, which, if not available on line, were requested and scanned through the UCD library. Titles and abstracts were imported into

\footnotetext{
${ }^{1}$ www.isiknowledge.com/

2 www.ncbi.nlm.nih.gov/pubmed/

${ }^{3}$ www.lib.ucdavis.edu
}

a reference manager system (EndNote, version X.02, Thomson Reuters, Carlsbad, CA, USA).

\subsection{Definition of parameters and predictors}

Stages of FMDV infection (Fig. 1) assessed here were the duration of the periods of latent, subclinically infectious, infectious, and incubation. Time-toevent information (days), where events were alternatively defined as the beginning and end of a given stage of FMDV infection (see Fig. 1), were recorded individually for animals reported on the assessed publication; for reports in which time-toevent was reported in hours, the value was divided by 24 to convert it into days and subsequently used as continuous values in the regression model, but rounded to fit a discrete distribution. Results from euthanized animals were considered censored records. First detection of FMDV $\left(t_{1}\right)$ was computed as time when virus was first detected, i.e. positive result, from a sampled tissue in an animal, following exposure to a donor $\left(t_{0}\right)$. Time from exposure $\left(t_{0}\right)$ to onset of clinical signs $\left(t_{2}\right)$, and last detection of FMDV $\left(t_{3}\right)$ were also computed (Fig. 1). Three stages of FMDV infection were fitted to parametric regression models: (1) incubation $\left(t_{0} \rightarrow t_{2}\right)$, (2) latent-and-subclinically infectious $\left(t_{0} \rightarrow t_{1} \rightarrow t_{2}\right)$, in which the incubation was categorized in latent and subclinical infectious using a dummy variable for each stage (latent, subclinical), and (3) duration of the infectiousness $\left(t_{1} \rightarrow t_{3}\right)$ that due to the limitation imposed by the duration of experiments, could not address the recurrent question about the carrier stage. Such definition of stages was necessary because of the different criteria for definition of parameters and reporting among experiments. Thus, although some papers reported only the duration of some of the stages, it was possible to infer additional parameters following the relationship by latent + subclinical $=$ incubation. For example, some experimental trials reported latent and incubation periods, but omitted the duration of the subclinical stage, which, following the approach described here, can be computed as the difference between the incubation and latent periods. Therefore, modeling latent and subclinical jointly provided more data than if they were assessed separately.

Seven factors were recorded that were hypothesized to influence the duration of FMDV-infection stages, namely, species of the susceptible and donor animals, route of infection, sampled tissue or secretion, clinical signs, virus topotype, and laboratory where the study was conducted (Tab. I). Experimental animals were grouped as cattle (bull, calf, steer, cattle, cow), pig, sheep (lamb, sheep), and goat. 
Table I. Epidemiological variables hypothesized to influence the duration of the stages of serotype $\mathrm{O}$ footand-mouth disease virus (FMDV) infection in an individual data meta-analysis of the peer reviewed literature.

\begin{tabular}{|c|c|c|}
\hline Variable & Description & Categories \\
\hline FMDV stage & Stages of FMDV infection & $\begin{array}{l}\text { Latent } \\
\text { Subclinical } \\
\text { Incubation } \\
\text { Infectious }\end{array}$ \\
\hline Experimental animal & $\begin{array}{l}\text { Type of animal exposed to } \\
\text { an infective donor via direct } \\
\text { or indirect contact }\end{array}$ & $\begin{array}{l}\text { Cattle } \\
\text { Pig } \\
\text { Small ruminants (sheep and goat) }\end{array}$ \\
\hline Donor species & $\begin{array}{l}\text { Species of the inoculated donor } \\
\text { compared to the experimental } \\
\text { animals exposed }\end{array}$ & $\begin{array}{l}\text { Same species } \\
\text { Different species }\end{array}$ \\
\hline $\begin{array}{l}\text { FMDV topotypes and } \\
\text { strains }\end{array}$ & $\begin{array}{l}\text { Topotypes and strains used } \\
\text { for the experiment }\end{array}$ & $\begin{array}{l}\text { Pan Asia: UKG 2001, } \\
\text { NET 2001, and Taiwan/97 } \\
\text { European strains of the } \\
\text { Euro-SA topotype: BFS } 1860 \text {, } \\
\text { and Brugge } \\
\text { MESA: Greece/94 } \\
\text { South American strains of the } \\
\text { Euro-SA topotype: Canefa, } \\
\text { and Campos }\end{array}$ \\
\hline Type of contact & $\begin{array}{l}\text { Type of contact between donors } \\
\text { and experimental animals }\end{array}$ & $\begin{array}{l}\text { Direct } \\
\text { Indirect }\end{array}$ \\
\hline Sample site & $\begin{array}{l}\text { Location in which the exposed } \\
\text { animal was sampled for } \\
\text { identification of the FMDV }\end{array}$ & $\begin{array}{l}\text { Sera (blood, serum) } \\
\text { Upper respiratory tract } \\
\text { (nasal swabs, oro-pharyngeal } \\
\text { fluids, saliva, pharynx, probang) } \\
\text { Excretion or secretion } \\
\text { (prepuce, urine, rectum, } \\
\text { feces, milk, semen, vagina) }\end{array}$ \\
\hline Clinical signs & Clinical signs reported & $\begin{array}{l}\text { Fever } \\
\text { First vesicles in mouth or feet } \\
\text { Generalized FMD infection }\end{array}$ \\
\hline FMD laboratory & $\begin{array}{l}\text { FMD reference laboratory } \\
\text { where the study was carried out }\end{array}$ & $\begin{array}{l}\text { Pirbright } \\
\text { Plum Island } \\
\text { Lelystad } \\
\text { PanAftosa }\end{array}$ \\
\hline
\end{tabular}

Because limited experimental evidence was available for goats, information from sheep and goat were grouped and analyzed in a single category referred to as small ruminants, and sensitivity of the results to this categorization was assessed by repeating the analyses maintaining the two original categories (sheep and goat). Categories of donor animals were dichotomized, depending on whether susceptible and donor animals were of the same or different species. Infection routes were categorized according to the source of FMDV. If transmission resulted from direct contact between an infected donor and susceptible animals that shared a common space or experimental unit (room), it was referred to as direct contact 
(DC); alternatively, if transmission resulted likely from a common airflow in which DC was not possible, it was referred as indirect contact (IC).

Tissues sampled were classified as serum, upper respiratory tract (nasal swabs, oropharyngeal fluids, saliva, pharynx, and probang), and either excretions or secretions (prepuce, urine, rectum, feces, milk, semen, and vagina). Clinical signs were categorized as unspecific (fever), primary signs of infection (first vesicles detected), and secondary or generalized signs of infection (vesicles detected in an organ other than the one in which primary signs of infection were detected). Virus strains were recorded and assessed according to the classification of topotypes shown in Table I and described elsewhere [37, 38].

FMD reference laboratories in which studies were carried out were the Institute for Animal Health (IAH, Pirbright, UK), the Plum Island Animal Disease Center (PIADC, New York, USA), the Central Institute for Animal Disease Control (CIDC, Lelystad, The Netherlands), and the Pan American Center for Foot-and-Mouth Disease (PanAftosa, Rio de Janeiro, Brazil).

\subsection{Collection and extraction of data}

Three randomly selected publications were reviewed independently by three authors of this paper. Report of results in those three publications varied from explicit tables and figures to narrative description of the values. Each of the three reviewers independently created a spreadsheet (Excel, Microsoft Corp., Redmond, WA, USA), in which each row represented an individual animal with a unique identification number (ID), and columns contained the corresponding values of duration of the stages of FMDV infection (in days) and of variables hypothesized to influence those values. Following such independent assessment, criteria for inclusion or exclusion of papers and a template for data collection were discussed and agreed upon by the authors. Subsequently, a double independent extraction of data from the publications was conducted by two of the authors. Extracted data were reviewed by four of the authors and in case of disagreement, a second revision of the manuscript was jointly conducted by the authors in order to reach consensus on the results interpretation.

\subsection{Statistical analyses of data distributions}

Fit of the distributions of data extracted for each FMDV-infection stage to probability density functions (PDF) commonly used in FMD simulation models $[6,13]$ was assessed. Using maximum likelihood estimates (MLE) for a maximum of 999 iterations, fitness of extracted data to 55 common theoretical distributions, including Pert, triangular, uniform, exponential, gamma, inverse Gaussian, log normal, log logistic, Pearson 5 and Weibull (continuous data) and Poisson (discrete data) was evaluated using two goodness of fit tests, Chi-square $\left(X^{2}\right)$ for discrete data, and Anderson-Darling for continuous data for a $p>0.05$ in either tests, meaning the distribution of the theoretical distribution were not significantly different from the distribution of the data. Theoretical distributions were formulated so that the probability for negative durations was null. Failure to reject the null hypothesis was assumed evidence that the data fitted the theoretical distribution. Descriptive statistics of the observed distributions of the FMD-infection stages, parameters of the theoretical distributions, and $p$ values for the goodness of fit tests were estimated using commercially available software, @RISK 5.0 (Palisade Inc., Newfield, NY, USA).

\subsection{Time-to-event models}

Three multivariate parametric time-to-event models were evaluated to identify sources of variation for the duration of FMD stages and control for potential confounders through a statistical screening step followed by a stepwise algorithm, which indicated confounding whenever the inclusion or exclusion of significant predictors resulted in a change in the final model coefficients of at least $20 \%$. First, duration of latent and subclinical periods was formulated in one model because those periods do not overlap and because they were clustered within a given animal. A second model was fitted for the duration of incubation; that second model included all the animals used in the previous model, following the relation latent + subclinical $=$ incubation, but also the data available from experimental animals in which only the incubation period was reported, and that for that reason, could not be included in the first model. A third model was constructed using infectious period as the response variable. These parametric regression models assumed that the baseline hazards function approximates a Weibull distribution, i.e. the hazard function for each period is restricted to be monotonically increasing (parameter $p>1$ ) or decreasing $(p<1)$ with time. The main advantage of the Weibull distribution is that it simultaneously behaves as proportional hazards $(\mathrm{PH})$ and accelerated failure time (AFT) regression models, so that relative event rates (HR) and relative extension of the 
time-to-event (event time ratio, TR) can be estimated, respectively. AFT models can be used to quantify the association between epidemiologic factors and the acceleration (shortening) or deceleration (delay) of the duration of FMDV-infection stages [14]. Survival data for the four stages of FMDV infection, i.e. latent-subclinical, incubation, and infectious were fitted and compared using the Akaike information criteria (AIC) statistic, which was defined as:

$$
\text { AIC }=-2 \log (\text { model likelihood })+2(k),
$$

where $k$ is the degrees of freedom of the model [14].

Explanatory variables were screened unconditionally, and variables for which associations at a significance level of $p<0.2$ were estimated, were considered candidate variables to fit a multivariate model. All biologically plausible two-way interactions between candidate variables were tested for the multivariate model and retained if $p<0.1$. Fitness of the final model was evaluated in a backward elimination process using a $p>0.05$ to remove variables that did not contribute significantly to the model. Two different frailty terms, which specify a function equivalent to a random effect in regression modeling, were evaluated in the models as a subject-parameter function. Frailty may happen because more than one value may have been computed for a single parameter in a single animal, e.g. if more than one tissue were sampled, which may result in different estimates of the duration of some of the stages assessed here. In this case, a frailty term was generated that referred to as multiple observations within the same subject. A second frailty term was evaluated to adjust for variability between experiments. These frailty terms were assumed to follow a gamma distribution [14], and if significant, only one of them was retained in the model by computing the AIC for both models that included and that did not include the frailty term. The likelihood ratio test for the frailty variance was calculated from the data using a stepwise elimination process $(p$ to enter $\leq 0.05$ and $p$ to exit $>0.10)[27,36]$. Data were analyzed using STATA 10.1 (STATA CorpLP, 2008). Goodness-of-fit of the model with the lowest AIC value was evaluated by visualization of the quantile-quantile (qq) plot of the times of survival percentiles. Approximation of the qq plot to a straight line was assumed to indicate a good fit of the model. Results were reported as the estimated baseline survival time (TR), which was computed as the exponential of the regression coefficient $(\beta)$ of significant variables in the fitted model.

\section{RESULTS}

\subsection{Literature search and study characteristics}

PubMed and ISI queries identified 103 and 145 references, respectively. Removal of duplicate and irrelevant articles resulted in a reference list of 55 publications retrieved and downloaded from the electronic searches. Inspection of the references listed in the 55 publications resulted in the identification of 26 additional references. From the resulting 81 publications, 62 articles were discarded because they contained information already reported elsewhere $(n=5)$, the original article could not be accessed $(n=7)$, results were irrelevant to our objective $(n=8)$, results were reported as group of animals (pooled results) $(n=7)$, results were not reported $(n=3)$, results presented information for other serotypes $(n=10)$, or methods used invasive routes (e.g., intradermal, intravenous) as source of FMDV infection $(n=22)$. Thus, 19 studies reported results of experimental trials for animals directly or indirectly exposed to animals infected with serotype O FMDV strains. The values of the duration of the stages of FMDV infection and of the epidemiological factors assessed here were extracted for 64 cattle, 149 sheep, 72 pigs, and 10 goats distributed in 20 experimental studies carried out in Pirbright $(n=13)$, Lelystad $(n=3)$, Plum Island $(n=3)$, and PanAftosa $(n=1)$ as shown in Table II.

\subsection{Descriptive statistics and fitted PDF distributions}

Descriptive statistics and distributions that best fit the stages of FMDV infection described here are presented in Figure 2 and Table III. Mean latent period ranged from 3.1 in pigs to 4.8 days in small ruminants. Mean subclinically infectious period ranged from to 2.0 in cattle to 2.3 days in pigs. Mean incubation period ranged from 5.6 to 6.6 days in pigs and small ruminants, respectively. Mean infectious period ranged from 3.3 in small ruminants to 5.7 days in pigs. 
Table II. Experimental studies included in the systematic review and individual data meta-analysis of the duration of infection stages of serotype O FMDV.

\begin{tabular}{|c|c|c|c|c|c|c|c|}
\hline \multirow[t]{2}{*}{ Author [ref.] } & \multirow{2}{*}{$\begin{array}{c}\text { FMD } \\
\text { laboratory }\end{array}$} & \multicolumn{4}{|c|}{ No. of animals } & \multirow{2}{*}{$\begin{array}{c}\text { No. of } \\
\text { observations } \\
(n=390)\end{array}$} & \multirow{2}{*}{$\begin{array}{l}\text { Topotype } \\
\text { (strains) }\end{array}$} \\
\hline & & $\begin{array}{c}\text { Cattle } \\
(n=64)\end{array}$ & $\begin{array}{c}\text { Pigs } \\
(n=72)\end{array}$ & $\begin{array}{c}\text { Small } \\
\text { ruminants } \\
(n=159)\end{array}$ & $\begin{array}{c}\text { Total } \\
(n=295)\end{array}$ & & \\
\hline Aggarwal et al. [1] & Pirbright & 3 & 4 & 8 & 15 & 15 & PanAsia (UKG 2001) \\
\hline Alexandersen et al. [3] & Pirbright & 0 & 8 & 0 & 8 & 8 & Euro - SA (BFS 1860) \\
\hline Bankowski et al. $^{\mathrm{a}}$ & Pirbright & 7 & 0 & 0 & 7 & 11 & PanAsia (UKG 2001) \\
\hline Blackwell et al. [8] & Plum Island & 6 & 0 & 0 & 6 & 17 & Euro - SA (Brugge) \\
\hline Burrows [10] & Pirbright & 12 & 10 & 9 & 31 & 31 & Euro - SA (BFS 1860) \\
\hline Burrows et al. [11] & Pirbright & 4 & 0 & 0 & 4 & 16 & Euro - SA (BFS 1860) \\
\hline Burrows et al. [12] & Pirbright & 2 & 0 & 0 & 2 & 3 & Euro - SA (BFS 1860) \\
\hline \multicolumn{8}{|l|}{ Donaldson and } \\
\hline Kitching [17] & Pirbright & 11 & 0 & 0 & 11 & 11 & Euro - SA (BFS 1860) \\
\hline Eble et al. [20] & Lelystad & 0 & 25 & 0 & 25 & 25 & PanAsia (Taiwan/97) \\
\hline Garland $^{\mathrm{b}}$ & Pirbright & 3 & 0 & 0 & 3 & 21 & Euro - SA (BFS 1860) \\
\hline \multicolumn{8}{|l|}{ Gibson and } \\
\hline Donaldson [23] & Pirbright & 0 & 0 & 19 & 19 & 31 & Euro - SA (BFS 1860) \\
\hline Gomes et al. [25] & PanAftosa & 4 & 0 & 0 & 4 & 8 & Euro - SA (Campos) \\
\hline Graves et al. [26] & Plum Island & 7 & 0 & 0 & 7 & 14 & Euro - SA (Canefa-2) \\
\hline Hughes et al. [29] & Pirbright & 0 & 0 & 64 & 64 & 64 & MESA (Greece/94) \\
\hline Hughes et al. [28] & Pirbright & 0 & 0 & 36 & 36 & 36 & MESA (Greece/94) \\
\hline \multicolumn{8}{|l|}{ McVicar and } \\
\hline Sutmoller [41] & Plum Island & 0 & 0 & 18 (10 goats) & 18 & 18 & Euro - SA (Canefa-2) \\
\hline Orsel et al. [42] & Lelystad & 0 & 0 & 5 & 5 & 5 & PanAsia (NET 2001) \\
\hline Orsel et al. [43] & Lelystad & 0 & 25 & 0 & 25 & 25 & PanAsia (NET 2001) \\
\hline Sellers et al. [49] & Pirbright & 4 & 0 & 0 & 4 & 24 & Euro - SA (BFS 1860) \\
\hline Zhang et al. [52] & Pirbright & 1 & 0 & 0 & 1 & 7 & PanAsia (UKG 2001) \\
\hline
\end{tabular}

a Bankowski B.M., Juleff N., Gibson D., Gloster J., Doel C., Cox S.J., Barnett P.V., Woolhouse M., Charleston B., Understanding FMDV transmission between cattle - preliminary data from animal experiments. Session of the Research Group of the Standing Technical Committee of European Commission for the Control of Foot-andMouth Disease (EUFMD) (2006) 165-175.

${ }^{\mathrm{b}}$ Garland, A.J.M., The inhibitory activity of secretions in cattle against FMDV, Ph.D. thesis, University of London, 1974, extracted from [2].

\subsection{AFT models}

Weibull distributions fit data to model the duration of the latent, subclinically infectious, incubation, and infectious periods (Tabs. IVVI). Inclusion of the shared frailty term for references and cluster of observations within subject, were statistically significant $(p<0.01)$ for the models of latent-subclinical and incubation, respectively. The point estimate of the Weibull shape parameter $(p)$ was $>1$ for the three models, indicating that the hazard functions for the duration of the periods assessed here increased monotonically with time; the value of $p$ was particularly large for the models of the incubation and infectiousness periods $(p \geq 3.5)$. An approximation to the $i$ th survival time for the duration of the FMD stages can be derived from the regression models using the following expression:

Duration of the stage (days) $=[-\ln (i$ th survival time $)]^{1 / p} \times\left[\exp \left(\beta_{0}+\beta_{i} \mathrm{x}_{i}+\ldots+\beta_{n} \mathrm{x}_{n}\right)\right]$, where $\beta_{0}$ is the intercept, and $\beta_{i}, \ldots, \beta_{n}$ are the coefficients for the $n$ predictor variables in the final model (Tabs. IV-VI). Due to restrictions of the statistical software used here, estimates using the equation are reported as a marginal estimate (unconditional) for animals with frailty of one, which corresponds to an additive random effect of zero. Hence, predictions are more 


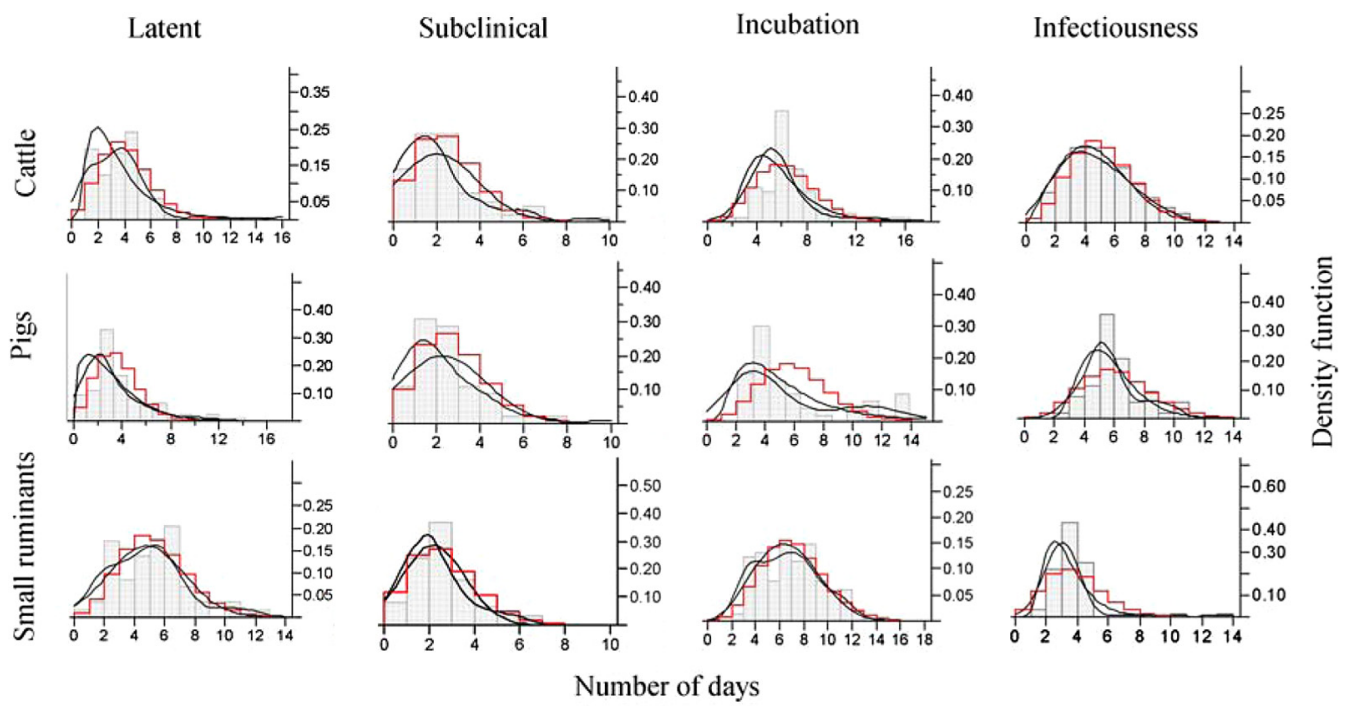

Figure 2. Frequency distributions and probability density functions fit to continuous (grey boxes) and discrete (red) data for experimental animals and FMD stage. Non-parametric density estimation using the kernel standard deviation (dashed line) was estimated for smoothing the distribution. (A color version of this figure is available at www.vetres.org.)

precise for the duration of the infectiousness, in which fitted regression model does not incorporate a frailty term, as it occurs for the latent-subclinical and incubation models. For example, to predict the median time of the infectious period in cattle detecting FMDV at the upper respiratory tract level would be:

$$
\begin{aligned}
\text { Infectiousness } t_{\mathrm{m}}= & {[-\ln (0.5)]^{1 / 3.54} } \\
& \times \exp (1.45+0.55) \\
\approx & 6.7 \text { days. }
\end{aligned}
$$

\subsubsection{Latent-subclinical period}

The regression model was fit using data collected from 154 animals in 15 experiments (Tab. IV). The estimated baseline survival time for both periods was 3.6 days $(95 \% \mathrm{CI}=3.07$, 4.31), from which the subclinically infectious period was 0.52 days shorter than the duration of the latent period. Survival time for the latent and subclinically infectious periods were about 0.53 days shorter in pigs than cattle. Duration of those periods was longer when presence of secondary clinical signs (generalized) other than nonspecific for FMDV infection was considered as the criterion to define the beginning of the clinical stage of infection. Two first order interaction terms, FMDV period by sample site and experimental animal by donor species, were retained in the multivariate model. The subclinically infectious period was 1.4 days longer when samples were collected from the upper respiratory tract, compared with samples collected from blood or any other secretion or excretion. In addition, the estimated baseline survival time for both periods was 2.5 days longer when pigs were exposed to infected donor of different species, compared to when they were exposed to donor pigs (same species). Inclusion of the FMD laboratory as predictor did not improve the fitness of the model.

\subsubsection{Incubation period}

Data of 221 animals from 17 experimental trials were used to fit a survival model that 
Table III. Descriptive statistics and distributions that best fit the stages of serotype $O$ foot-and-mouth disease virus (FMDV) infection in an individual data meta-analysis exercise. Data were fit using equal width intervals for continuous and discrete data (Poisson) distributions. Bolded letters indicates a $p>0.05$ value, which indicates the theoretical distribution fit the data well.

\begin{tabular}{lccccc}
\hline FMD stage & $\begin{array}{c}\text { Animal } \\
\text { species }\end{array}$ & No. & $\begin{array}{c}\text { Mean, } \\
\text { median } \\
(25 \text { th, } 75 \text { th } \\
\text { percentile })\end{array}$ & $\begin{array}{c}\text { Distribution } \\
\text { (parameters) }\end{array}$ & Poisson $(\lambda)$ \\
\hline Latent & Cattle & 136 & $3.6,3(2,5)$ & Weibull $(\alpha=1.782, \beta=3.974)$ & 3.59 \\
& Pig & 72 & $3.1,2(2,4)$ & Gamma $(\alpha=1.617, \beta=1.914)$ & 3.07 \\
& Small ruminant & 58 & $4.8,5(3,6)$ & Pert $(m=3.963, a=0, b=13.983)$ & 4.79 \\
Subclinical & Cattle & 119 & $2.0,2(1,3)$ & Gamma $(\alpha=1.222, \beta=1.672)$ & 2.04 \\
& Pig & 45 & $2.3,2(1,3)$ & Inverse Gaussian $(\mu=2.3, \lambda=3.045)$ & 2.27 \\
& Small ruminant & 62 & $2.2,2(1,3)$ & Gamma $(\alpha=2.4, \beta=0.898)$ & 2.16 \\
Incubation & Cattle & 59 & $5.9,5(5,6)$ & Log logistic $(\gamma=0, \beta=5.3, \alpha=4.02)$ & $\mathbf{5 . 9}$ \\
& Pig & 46 & $5.6,4(3,9)$ & Pearson $5(\alpha=3.05, \beta=11.72)$ & 5.58 \\
& Small ruminant & 128 & $6.6,6(4,8)$ & Weibull $(\alpha=2.784, \beta=7.426)$ & $\mathbf{6 . 5 9}$ \\
& Cattle & 71 & $4.4,4(3,6)$ & Gamma $(\alpha=3.969, \beta=1.107)$ & $\mathbf{4 . 3 9}$ \\
& Pig & 53 & $5.7,5(5,6)$ & Log logistic $(\gamma=0, \beta=5.39, \alpha=5.474)$ & 5.69 \\
& Small ruminant & 59 & $3.3,3(2,4)$ & Pearson $5(\alpha=6.188, \beta=17.192)$ & 3.32 \\
\hline
\end{tabular}

Table IV. Weibull model (shape parameter $p=1.72$ ) fitted for the duration of the latent and subclinical periods, based on FMDV-transmission experiments from 154 animals in 15 experimental trials conducted between 1967 and 2007.

\begin{tabular}{|c|c|c|c|c|c|}
\hline Variable & Category & Time ratio & Coefficient $(\beta)$ & $95 \% \mathrm{CI}$ of $\beta$ & $p$ value \\
\hline FMDV stage & $\begin{array}{c}\text { Latent } \\
\text { Subclinical }\end{array}$ & 0.52 & -0.65 & $-0.79,-0.50$ & $<0.01$ \\
\hline $\begin{array}{l}\text { Experimental } \\
\text { animal }\end{array}$ & $\begin{array}{l}\text { Cattle } \\
\text { Pig }\end{array}$ & 0.53 & -0.63 & $-1.03,-0.23$ & $<0.01$ \\
\hline Clinical signs & $\begin{array}{c}\text { Fever } \\
\text { Generalized }\end{array}$ & 1.26 & 0.23 & $0.02,0.45$ & 0.03 \\
\hline $\begin{array}{l}\text { Interaction terms } \\
\text { FMDV stage } \times \\
\text { sample site }\end{array}$ & $\begin{array}{l}\text { Subclinical sampled } \\
\text { from upper } \\
\text { respiratory tract }\end{array}$ & 1.41 & 0.34 & $0.17,0.52$ & $<0.01$ \\
\hline $\begin{array}{l}\text { Experimental } \\
\text { animal } \times \text { donor specie }\end{array}$ & $\begin{array}{l}\text { Pigs exposed to a } \\
\text { different specie } \\
\text { Intercept }\end{array}$ & 2.53 & $\begin{array}{l}0.93 \\
1.29\end{array}$ & $\begin{array}{l}0.46,1.40 \\
1.12,1.46\end{array}$ & $\begin{array}{l}<0.01 \\
<0.01\end{array}$ \\
\hline
\end{tabular}

assumed a Weibull distribution for the incubation period (Tab. V). Baseline survival time for the incubation period was estimated to be 2.9 days $(95 \% \mathrm{CI}=2.6,3.3)$ and the final model included a statistically significant $(p<0.01)$ shared frailty term for the within subject cluster. The baseline survival time for the incubation period for small ruminants was 2.3 days longer than for cattle and significantly $(p<0.01)$ longer when the experimental design 
Table V. Weibull model (shape parameter $p=5.14$ ) fitted for the duration of the incubation period, based on FMDV-transmission experiments using 221 animals in 17 trials conducted between 1967 and 2007.

\begin{tabular}{|c|c|c|c|c|c|}
\hline Variable & Category & Time ratio & Coefficient $(\beta)$ & $95 \% \mathrm{CI}$ of $\beta$ & $p$ value \\
\hline $\begin{array}{l}\text { Experimental } \\
\text { animal }\end{array}$ & $\begin{array}{c}\text { Cattle } \\
\text { Small ruminants }\end{array}$ & 2.32 & 0.84 & $0.71,0.97$ & $<0.01$ \\
\hline Type of contact & $\begin{array}{l}\text { Direct } \\
\text { Indirect }\end{array}$ & 1.62 & 0.48 & $0.29,0.68$ & $<0.01$ \\
\hline Donor specie & $\begin{array}{c}\text { Same specie } \\
\text { Different specie }\end{array}$ & 1.86 & 0.62 & $0.45,0.78$ & $<0.01$ \\
\hline FMDV topotype & $\begin{array}{c}\text { Pan Asia } \\
\text { Euro-SA (European strains) } \\
\text { Euro - SA } \\
\text { (South American strains) }\end{array}$ & $\begin{array}{l}2.12 \\
5.16\end{array}$ & $\begin{array}{l}0.75 \\
1.64\end{array}$ & $\begin{array}{l}0.58,0.91 \\
1.25,2.03\end{array}$ & $\begin{array}{l}<0.01 \\
<0.01\end{array}$ \\
\hline $\begin{array}{l}\text { Clinical signs } \\
\text { FMD laboratory }\end{array}$ & $\begin{array}{l}\text { Fever } \\
\text { Generalized } \\
\text { Pirbright }\end{array}$ & 1.28 & 0.25 & $0.13,0.37$ & $<0.01$ \\
\hline & $\begin{array}{l}\text { Plum Island } \\
\text { PanAftosa }\end{array}$ & $\begin{array}{l}0.31 \\
0.16\end{array}$ & $\begin{array}{l}-1.17 \\
-1.83\end{array}$ & $\begin{array}{l}-1.5,-0.85 \\
-2.36,-1.3\end{array}$ & $\begin{array}{l}<0.01 \\
<0.01\end{array}$ \\
\hline $\begin{array}{l}\text { Interaction terms } \\
\text { Experimental animal } \times\end{array}$ & Small ruminants exposed & 0.35 & -1.06 & $-1.25,-0.87$ & $<0.01$ \\
\hline $\begin{array}{l}\text { Type of contact } x \\
\text { topotype }\end{array}$ & $\begin{array}{c}\text { Indirect } \times \text { Euro }- \text { SA } \\
\text { (European strains) }\end{array}$ & 0.25 & -1.4 & $-1.62,-1.17$ & $<0.01$ \\
\hline & Intercept & & 1.07 & $0.96,1.18$ & $<0.01$ \\
\hline
\end{tabular}

included indirect contact, contact with a different donor species, and use of Euro-SA topotype strains, and when signs of generalization of FMDV infection were used to define the end of the period. The incubation period was shorter for experiments conducted at Plum Island and PanAftosa, compared with results from experiments conducted at Pirbright. Two interaction terms improved the model fitness. The incubation period was approximately 0.35 days shorter for small ruminants exposed to pigs or cattle, compared with those exposed to sheep. In addition, the period was 0.25 days shorter when the experiment was based on indirect contact with animals infected with Brugge or BFS 1860 strains (Euro-SA topotype), compared with results when with animals were infected via indirect contact with Pan Asia topotype strains (Tab. V).

\subsubsection{Infectiousness period}

Duration of the infectious period was fit to a Weibull model using data collected from 138 animals in 10 different trials (Tab. VI). The baseline duration of the infectious period was 4.3 days $(95 \% \mathrm{CI}=3.9,4.7)$. Duration of the infectious period was approximately 1.5 days longer in small ruminants compared with cattle, and 0.58-0.46 days shorter when Greece/94, Canefa, and Campos strains were used, compared with Pan Asia strains, respectively. Duration of the infectious period was 1.7 days longer samples were collected from the upper respiratory tract, compared with collection of samples from blood or from any other secretion or excretion. The infectious period was shorter for experiments conducted at Lelystad compared with those carried out at Pirbright. 
Table VI. Weibull model (shape parameter $p=3.54$ ) fitted for the period of infectiousness, based on FMDV-transmission experiments using 138 animals in 10 trials conducted between 1967 and 2007.

\begin{tabular}{lccccc}
\hline Variable & Category & Time ratio & Coefficient $(\beta)$ & $95 \%$ CI of $\beta$ & $p$ value \\
\hline Experimental animal & $\begin{array}{c}\text { Cattle } \\
\text { Small ruminants }\end{array}$ & 1.49 & 0.4 & $0.25,0.55$ & $<0.01$ \\
FMDV topotype & $\begin{array}{c}\text { Pan Asia } \\
\text { MESA }\end{array}$ & 0.46 & -0.77 & $-0.96,-0.58$ & $<0.01$ \\
& Euro - SA (South American strains) & 0.58 & -0.55 & $-0.68,-0.41$ & $<0.01$ \\
Sample site & $\quad$ Sera & & & & \\
& Upper respiratory tract & 1.73 & 0.55 & $0.43,0.68$ & $<0.01$ \\
FMD laboratory & Pirbright & & & & \\
& Lelystad & 0.17 & -1.8 & $-0.31,-0.05$ & $<0.01$ \\
& Intercept & & 1.45 & $1.35,1.55$ & $<0.01$ \\
\hline
\end{tabular}

No interaction or frailty terms improved the model fitness.

\section{DISCUSSION}

A systematic review and meta-analysis of experimental trials conducted at four FMD reference laboratories from 1960 through 2007 was used here to estimate species-specific probability functions of the duration of the latency, incubation, subclinically infectious, and infectiousness periods of FMD infection, and the association of those functions with epidemiological factors. This information will help to improve the precision of simulation models and, ultimately, the ability of countries to prevent and control FMD epidemics.

Although the selection criteria imposed to conduct the meta-analysis dismissed an important number of studies, for example, reporting pooled results $(n=7)$, the method used here offers certain advantages compared to traditional meta-analysis techniques used elsewhere [39]. Meta-analyses are conducted using information provided by a series of studies to produce a point estimate of an effect and measures of the precision of such estimate [47, 48]. Conversely, in the study here, data was extracted on an individual basis through a systematic review process, which provides and makes use of more information than traditional meta-analysis techniques that are typically applied to aggregated data [39]. For example, when data are identified and collected on an individual basis, rather than grouped, quality of data entry is easy to verify and outliers can be identified, and potentially removed. Moreover, different subgroup of records, such as type of experiment or FMD laboratory in which the study was conducted, can be created and the extent of the influence of such attributes on the value of the outcome may be assessed. Also, a large variety of techniques may be used to report the outcome of the analysis, including probability distributions or time to occurrence of the event. In the study here, for example, parametric regression models were used to quantify the time to occurrence of specific events that indicate a change in the stage of FMD infection, which could not have been estimated if aggregated data were used in the analyses.

Results reported here were consistent with previous knowledge about FMD pathogenesis, and its epidemiology. Mostly, probability distributions were left-skewed (Fig. 2), as observed in many survival data, and duration of each stage of FMD-infection varied with the experimental animal species assessed (Tabs. III-VI).

A standard approach in disease modeling is to fit parametric distributions, such as those presented here, to capture the variability of specific parameters of the model. However, fitted 
distributions represent a mixture of all the experimental conditions included in the dataset. The regression models fitted here show that specific experimental conditions heavily influence the duration of the studied FMDV stages. For example, mean duration of the infectiousness in small ruminants seemed to be shorter than in cattle, as suggested by the descriptive distributions of the experimental data (Tab. III). However, adjustment of the parameter by the value of significant variables suggests that, in average, duration of infectiousness is longer in small ruminants, compared to cattle (Tab. VI). Consequently, the PDF represent descriptive approximations of the distribution of the empirical data but their use to parameterize stochastic models without taking into account the influence of host or agent factors may bias the results of an epidemiological model. Alternatively, we provide an adjusted approach using parametric regression models that modelers can use to parameterize, using the coefficients from significant variables, simulation models. Fitted models can be used to approximate the value of specific parameters adjusted by the value of significant variables $[27,36]$, and 6 tables with a mixture of combinations of predictions for the duration of FMD stages are provided (on line Supplementary data available at www.vetres.org).

In general, susceptible animals that were exposed to an FMD-infected animal from a different species showed longer incubation periods than susceptible animals exposed to infected animals from the same species. Because incubation was computed here as the time between exposure to an infected animal and the onset of clinical signs there are many factors that may have influenced such finding. One possible explanation is that animals from the same species are expected to interact with each other more frequently than animals from a different species, which may result in largest number of effective contacts. Another possible explanation may be the influence of species-specific differences in the pathogenesis of the disease. For example, duration of latency and duration of the subclinical period was longest for pigs exposed to cattle or small ruminants, resulting in longest duration of the incubation period, which may be explained by lowest susceptibility of pigs to aerosol infection [2]. Small ruminants showed clinical signs faster after exposure to pigs compared to exposure to other small ruminants, which may be due to a combination of highest susceptibility of sheep to aerosol infection [18] and large amount of FMDV excreted by pigs $[15,16,50]$. Another possible explanation for the observed longer incubation for susceptible animals exposed to same species-infected animals compared to those exposed to different species-infected animals may be due to differences in the serotype- or strain-specific susceptibility of species. For example, certain serotypes and strains may be more adapted to some animal species than another as suggested by the high correlation between strain and experimental animal species (Tab. II).

Duration of latent-subclinical and incubation periods was longer for small ruminants compared to cattle. Such pattern is consistent with previous knowledge on the epidemiology $[9,44]$, and pathogenesis of the disease in small ruminants $[22,40]$, which, under certain conditions, are considered responsible for the spread of FMDV due to the relatively mild signs of disease that the virus produces in those species. Because, compared to field conditions, detection of clinical signs is easier in controlled experimental trials, it is possible that the delay in the appearance of clinical signs reported here may also be associated with mild signs of disease and, ultimately, with the misdiagnosis of FMD infection under field conditions [21], in which individual animal observation is less frequent and less detailed.

Results of this study also indicate that duration of the stages of FMDV infection are heavily dependent on the specific virus strain associated with the infection, as suggested by the influence that virus topotypes had as predictors of the final models. For example, infection with Canefa and Campos strains resulted in significantly longer incubation periods compared to infection with the Pan Asia strain. This finding is consistent with field observations that indicate that some emergent sublineages of Pan Asia strains have increased virulence for particular species [19] and there 
are genomic changes that contribute to this adaptation [46], which can explain in part, the virus replacement observed in certain regions of the world. Although such finding is biologically sound and it is consistent with previous field observations, which indicate that certain strains are more virulent than others for specific susceptible populations $[38,45,46]$, straindependent factors, are commonly ignored in FMD-transmission and spread models. Therefore, results suggest that accuracy of FMD models would be benefited if strain-dependent factors, such as fitness to the host, competence, adaptation, and evolution, were considered for the parameterization of FMD spread.

Results were also influenced by the type of sampled tissue, suggesting that sampling from the upper respiratory tract may increase the probability of early detection of FMDV. This result suggests that air samplers and similar pen-side tests and devices may contribute to reduce time to detection of FMDV infection and become a key component of near real time surveillance systems.

In conclusion, the study here represents the first quantitative assessment of the nature and extent of the association between lengths of each stage of FMDV infection and strain and host specific factors. Results presented here can be used to improve the accuracy of FMD transmission and spread models, and ultimately, the ability of countries to prevent and early control FMDV incursions.

Acknowledgements. This study was supported in part by the U.S. National Center for Medical Intelligence and the Department of Homeland Security and the National Center for Foreign Animal and Zoonotic Disease Defense.

\section{REFERENCES}

[1] Aggarwal N., Zhang Z., Cox S., Statham R., Alexandersen S., Kitching R.P., Barnett P.V., Experimental studies with foot-and-mouth disease virus, strain $\mathrm{O}$, responsible for the 2001 epidemic in the United Kingdom, Vaccine (2002) 20:2508-2515.

[2] Alexandersen S., Donaldson A.I., Further studies to quantify the dose of natural aerosols of foot-and-mouth disease virus for pigs, Epidemiol. Infect. (2002) 128:313-323.
[3] Alexandersen S., Quan M., Murphy C., Knight J., Zhang Z., Studies of quantitative parameters of virus excretion and transmission in pigs and cattle experimentally infected with foot-and-mouth disease virus, J. Comp. Pathol. (2003) 129:268-282.

[4] Anderson R.M., May R.M., Infectious diseases of humans: dynamics and control, Oxford University Press, New York, 1991, pp. 27-86.

[5] Bates T.W., Thurmond M.C., Carpenter T.E., Direct and indirect contact rates among beef, dairy, goat, sheep, and swine herds in three California counties, with reference to control of potential footand-mouth disease transmission, Am. J. Vet. Res. (2001) 62:1121-1129.

[6] Bates T.W., Thurmond M.C., Carpenter T.E., Description of an epidemic simulation model for use in evaluating strategies to control an outbreak of footand-mouth disease, Am. J. Vet. Res. (2003) 64: 195-204.

[7] Belsham G.J., Distinctive features of foot-andmouth disease virus, a member of the picornavirus family; aspects of virus protein synthesis, protein processing and structure, Prog. Biophys. Mol. Biol. (1993) 60:241-260.

[8] Blackwell J.H., McKercher P.D., Kosikowski F.V., Carmichael L.E., Gorewit R.C., Concentration of foot-and-mouth disease virus in milk of cows infected under simulated field conditions, J. Dairy Sci. (1982) 65:1624-1631.

[9] Branscum A.J., Perez A.M., Johnson W.O., Thurmond M.C., Bayesian spatiotemporal analysis of foot-and-mouth disease data from the Republic of Turkey, Epidemiol. Infect. (2008) 136:833-842.

[10] Burrows R., Excretion of foot-and-mouth disease virus prior to the development of lesions, Vet. Rec. (1968) 82:387-388.

[11] Burrows R., Mann J.A., Greig A., Chapman W.G., Goodridge D., The growth and persistence of footand-mouth disease virus in the bovine mammary gland, J. Hyg. (Lond.) (1971) 69:307-321.

[12] Burrows R., Mann J.A., Garland A.J., Greig A., Goodridge D., The pathogenesis of natural and simulated natural foot-and-mouth disease infection in cattle, J. Comp. Pathol. (1981) 91:599-609.

[13] Carpenter T.E., Thurmond M.C., Bates T.W., A simulation model of intraherd transmission of foot and mouth disease with reference to disease spread before and after clinical diagnosis, J. Vet. Diagn. Invest. (2004) 16:11-16.

[14] Cleves M.A., Gould W.W., Gutierrez R.G., An introduction to survival analysis using Stata, Stata Press, Florence, 2008, pp. 197-292. 
[15] Donaldson A.I., Herniman K.A., Parker J., Sellers R.F., Further investigations on the airborne excretion of foot-and-mouth disease virus, J. Hyg. (Lond.) (1970) 68:557-564.

[16] Donaldson A.I., Foot-and-mouth disease: the principal features, Irish Vet. J. (1987) 41:325-327.

[17] Donaldson A.I., Kitching R.P., Transmission of foot-and-mouth disease by vaccinated cattle following natural challenge, Res. Vet. Sci. (1989) 46:9-14.

[18] Donaldson A.I., Alexandersen S., Sorensen J.H., Mikkelsen T., Relative risks of the uncontrollable (airborne) spread of FMD by different species, Vet. Rec. (2001) 148:602-604.

[19] Dunn C.S., Donaldson A.I., Natural adaption to pigs of a Taiwanese isolate of foot-and-mouth disease virus, Vet. Rec. (1997) 141:174-175.

[20] Eble P., de Koeijer A., Bouma A., Stegeman A., Dekker A., Quantification of within- and between-pen transmission of foot-and-mouth disease virus in pigs, Vet. Res. (2006) 37:647-654.

[21] Geering W.A., Foot-and-mouth disease in sheep, Aust. Vet. J. (1967) 43:485-488.

[22] Gibbens J.C., Sharpe C.E., Wilesmith J.W., Mansley L.M., Michalopoulou E., Ryan J.B., Hudson M., Descriptive epidemiology of the 2001 foot-and-mouth disease epidemic in Great Britain: the first five months, Vet. Rec. (2001) 149:729-743.

[23] Gibson C.F., Donaldson A.I., Exposure of sheep to natural aerosols of foot-and-mouth disease virus, Res. Vet. Sci. (1986) 41:45-49.

[24] Gloster J., Freshwater A., Sellers R.F., Alexandersen S., Re-assessing the likelihood of airborne spread of foot-and-mouth disease at the start of the 1967-1968 UK foot-and-mouth disease epidemic, Epidemiol. Infect. (2005) 133:767-783.

[25] Gomes I., Ramalho A.K., de Mello P.A., Infectivity assays of foot-and-mouth disease virus: contact transmission between cattle and buffalo (Bubalus bubalis) in the early stages of infection, Vet. Rec. (1997) 140:43-47.

[26] Graves J.H., McVicar J.W., Sutmoller P., Trautman R., Contact transmission of foot-and-mouth disease from infected to susceptible cattle, J. Infect. Dis. (1971) 123:386-391.

[27] Gutierrez R.G., Parametric frailty and shared frailty survival models, Stat. J. (2002) 2:22-44.

[28] Hughes G.J., Mioulet V., Haydon D.T., Kitching R.P., Donaldson A.I., Woolhouse M.E., Serial passage of foot-and-mouth disease virus in sheep reveals declining levels of viraemia over time, J. Gen. Virol. (2002) 83:1907-1914.

[29] Hughes G.J., Mioulet V., Kitching R.P., Woolhouse M.E., Alexandersen S., Donaldson A.I. Foot-and-mouth disease virus infection of sheep: implications for diagnosis and control, Vet. Rec. (2002) 150:724-727.

[30] James A.D., Rushton J., The economics of foot and mouth disease, Rev. Sci. Tech. Off. Int. Epizoot. (2002) 21:637-644.

[31] Keeling M.J., Woolhouse M.E., Shaw D.J., Matthews L., Chase-Topping M., Haydon D.T., et al., Dynamics of the 2001 UK foot and mouth epidemic: stochastic dispersal in a heterogeneous landscape, Science (2001) 294:813-817.

[32] Keeling M.J., Models of foot-and-mouth disease, Proc. Biol. Sci. (2005) 272:1195-1202.

[33] Kitching R.P., Clinical variation in foot and mouth disease: cattle, Rev. Sci. Tech. Off. Int. Epizoot. (2002) 21:499-504.

[34] Kitching R.P., Alexandersen S., Clinical variation in foot and mouth disease: pigs, Rev. Sci. Tech. Off. Int. Epizoot. (2002) 21:513-518.

[35] Kitching R.P., Hughes G.J., Clinical variation in foot and mouth disease: sheep and goats, Rev. Sci. Tech. Off. Int. Epizoot. (2002) 21:505-512.

[36] Kleinbaum D.G., Klein M., Survival analysis: a self-learning text, Springer, New York, 2005.

[37] Knowles N.J., Samuel A.R., Molecular epidemiology of foot-and-mouth disease virus, Virus Res. (2003) 91:65-80.

[38] Knowles N.J., Samuel A.R., Davies P.R., Midgley R.J., Valarcher J.F., Pandemic strain of footand-mouth disease virus serotype $\mathrm{O}$, Emerg. Infect. Dis. (2005) 11:1887-1893.

[39] Lean I.J., Rabiee A.R., Duffield T.F., Dohoo I.R., Invited review: use of meta-analysis in animal health and reproduction: methods and applications, J. Dairy Sci. (2009) 92:3545-3565.

[40] Mansley L.M., Dunlop P.J., Whiteside S.M., Smith R.G., Early dissemination of foot-and-mouth disease virus through sheep marketing in February 2001, Vet. Rec. (2003) 153:43-50.

[41] McVicar J.W., Sutmoller P., Experimental foot-and-mouth disease in sheep and goats: an epizootiological model, Arch. Gesamte Virusforsch (1972) 38:85-96.

[42] Orsel K., Dekker A., Bouma A., Stegeman J.A., de Jong M.C., Quantification of foot and mouth disease virus excretion and transmission within groups 
of lambs with and without vaccination, Vaccine (2007) 25:2673-2679.

[43] Orsel K., de Jong M.C., Bouma A., Stegeman J.A., Dekker A., Foot and mouth disease virus transmission among vaccinated pigs after exposure to virus shedding pigs, Vaccine (2007) 25:6381-6391.

[44] Perez A.M., Thurmond M.C., Grant P.W., Carpenter T.E., Use of the scan statistic on disaggregated province-based data: foot-and-mouth disease in Iran, Prev. Vet. Med. (2005) 71:197-207.

[45] Samuel A.R., Knowles N.J., Foot-and-mouth disease type $\mathrm{O}$ viruses exhibit genetically and geographically distinct evolutionary lineages (topotypes), J. Gen. Virol. (2001) 82:609-621.

[46] Samuel A.R., Knowles N.J., Foot-and-mouth disease virus: cause of the recent crisis for the UK livestock industry, Trends Genet. (2001) 17:421-424.

[47] Sanchez J., Dohoo I., Carrier J., DesCoteaux L., A meta-analysis of the milk-production response after anthelmintic treatment in naturally infected adult dairy cows, Prev. Vet. Med. (2004) 63:237-256.
[48] Sanchez J., Dohoo I.R., Christensen J., Rajic A., Factors influencing the prevalence of Salmonella spp. in swine farms: a meta-analysis approach, Prev. Vet. Med. (2007) 81:148-177.

[49] Sellers R.F., Burrows R., Mann J.A., Dawe P., Recovery of virus from bulls affected with foot-andmouth disease, Vet. Rec. (1968) 83:303.

[50] Sellers R.F., Parker J., Airborne excretion of foot-and-mouth disease virus, J. Hyg. (Lond.) (1969) 67:671-677.

[51] Ward M.P., Highfield L.D., Vongseng P., Graeme Garner M., Simulation of foot-and-mouth disease spread within an integrated livestock system in Texas, USA, Prev. Vet. Med. (2009) 88:286-297.

[52] Zhang Z., Murphy C., Quan M., Knight J., Alexandersen S., Extent of reduction of foot-andmouth disease virus RNA load in oesophagealpharyngeal fluid after peak levels may be a critical determinant of virus persistence in infected cattle, J. Gen. Virol. (2004) 85:415-421. 